MACROPHAGES

\section{Immune evasion by anthrax}

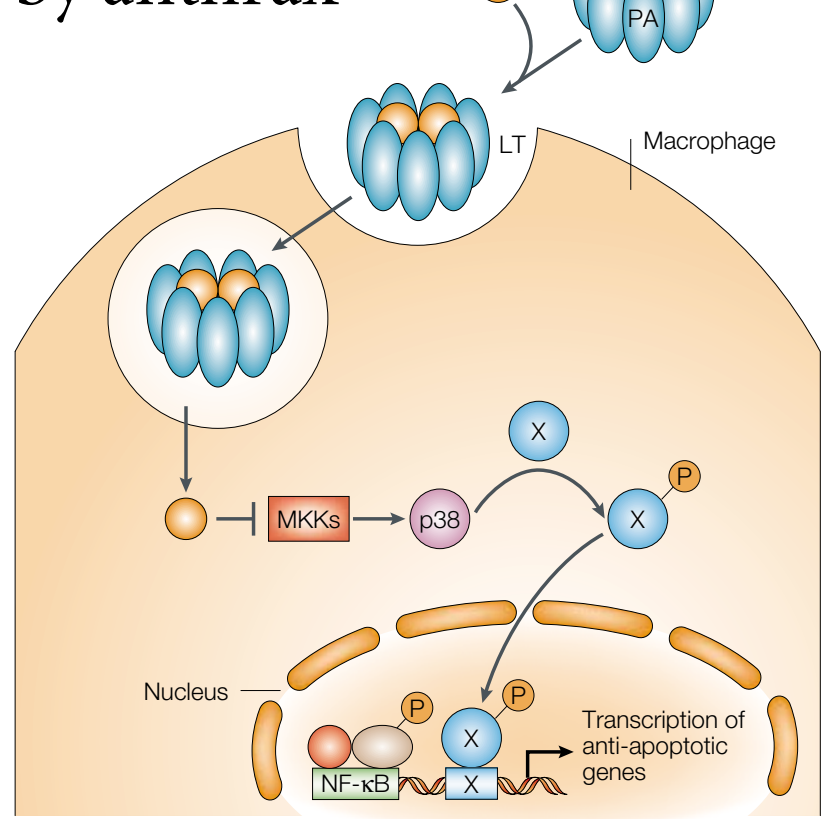

Bacillus anthracis, the causative agent of anthrax, kills macrophages and evades the host immune response by secreting a protein that leads to the inhibition of p38 mitogen-activated protein kinase (p38 MAPK), according to a report in Science. The activity of this MAPK is required for the transcription of certain downstream nuclear factor- $\mathrm{\kappa B}(\mathrm{NF}-\kappa \mathrm{B})$ targets.

Three proteins secreted by $B$. anthracis are important for its pathogenicity: protective antigen $(\mathrm{PA})$, oedema factor (EF) and lethal factor (LF). PA enables EF and LF to enter the cytosol of host cells, where EF causes tissue oedema and LF acts as a metalloprotease, cleaving MAPK kinases (MKKs) and thereby preventing MAPK activation. Lethal toxin (LT; a complex of activated PA and LF) has been shown to be cytotoxic for macrophages, but it was not known how the blockade of MAPK signalling tied in with these cytotoxic effects.

Park and colleagues carried out a series of in vitro experiments to study the effects of LT on lipopolysaccharide
(LPS)-activated macrophages. First, they showed that LT causes the apoptosis of LPS-treated macrophages; then, they investigated the signalling pathways involved. At the concentration that is required to induce apoptosis, LT inhibits the activation of ERK, c-Jun $\mathrm{NH}_{2}$-terminal kinase 1 (JNK1) and $\mathrm{p} 38$, but the activity of JNK2 and inhibitor of NF-KB (IKB) kinase (IKK) is unaffected. The authors used specific MAPK inhibitors to determine the contribution of these MAPK cascades to LT-induced apoptosis. Only the $\mathrm{p} 38$-specific inhibitor (SB202190) induced apoptosis of LPS-activated macrophages, which indicates that LT might cause apoptosis by inhibiting p38 signalling.

Macrophages lacking IKK and NF- $\kappa B$ activity were shown to be sensitive to LPS-induced apoptosis also. So, Park et al. investigated whether p38 synergizes with NF- $\kappa B$ to induce the transcription of anti-apoptotic genes. They used real-time PCR to study the effects of blocking p 38 signalling on the expression of known

\section{Express yourself}

Accumulating evidence indicates that the ectopic expression of tissue-specific proteins by rare thymic medullary epithelial cells (MECs) might be important for the deletion of potentially harmful $\mathrm{T}$ cells during their development (see the Highlight 'Random reflections' in our December 2001 issue). Now, clues from a human genetic disorder have led Harvard researchers Mark Anderson and colleagues to suggest a potential mechanism for this ectopic gene expression.

In autoimmune polyendocrine syndrome type 1 (APS1), loss-of-function mutations in a single gene, AIRE (autoimmune regulator), which encodes a transcriptional activator, result in autoimmune responses to many tissues and organs. Intriguingly, AIRE is expressed most highly in the thymus, specifically by MECs. So, does AIRE have a role in ectopic gene expression by MECs, leading to tolerance induction?

Aire-deficient mice have a similar autoimmune syndrome to patients with APS1, but an earlier study concluded that this was the result of a defect in peripheral tolerance. Anderson and colleagues, who report their findings in Science, generated their own Aire-knockout mice and made radiation chimeras to investigate this issue further.

They show that to prevent autoimmunity, Aire must be expressed by radio-resistant, non-haematopoietic cells. But, are these cells in the thymus or in the periphery? Realtime reverse-transcriptase-PCR did not detect expression of Aire in parenchymal tissues. However, low levels of expression were found in peripheral lymphoid tissues, although at much lower levels than in the thymus. A thymic-graft experiment was used to settle the issue. Thymi from Aire $^{-/-}$or $\mathrm{Aire}^{+/+}$mice were depleted of haematopoietic cells then transplanted into athymic recipients. Only recipients of Airedeficient thymi developed autoantibodies, which indicates that Aire must be expressed by thymic stromal cells to prevent autoimmunity.

So, is Aire involved in the ectopic expression of organ-specific antigens by MECs? Microarrays were used to compare the expression of 12,000 mouse genes by Aire $^{-l-}$ and Aire ${ }^{+/+}$MECs. The absence of Aire resulted in the decreased expression of 100-300 genes. Strikingly, the 30 genes that were downregulated to the greatest extent encode mainly tissue-specific proteins, several of which are known to be targets of autoimmunity in patients with APS1, such as insulin and cytochrome $\mathrm{P} 450$.

This study provides the first insight into the molecular basis of the phenomenon of promiscuous gene expression by MECs. However, at present, only a correlation between reduced levels of messenger RNA and autoimmunity has been observed - we do not know how the lower levels of mRNA influence protein expression, antigen presentation, T-cell repertoire selection and immune responsiveness. There is also the question of how AIRE might regulate the ectopic expression of such a large, but select, group of genes. These are important questions for future investigation.

Jennifer Bell

(9) References and links ORIGINAL RESEARCH PAPER Anderson, M. S. et al. Projection of an immunological self-shadow within the thymus by the Aire protein. Science 10 October 2002 (DOI: 10.1126/science. 1075958)

FURTHER READING Derbinski, J., Schulte, A., Kyewski, B. \& Klein, L. Promiscuous gene expression in medullary thymic epithelial cells mirrors the peripheral self. Nature Immunol. 2, 1032-1039 (2001) | Klein, L. \& Kyewski, B. 'Promiscuous' expression of tissue antigens in the thymus: a key to T-cell tolerance and autoimmunity? J. Mol. Med. 78, 483-494 (2000)

\section{WEB SITE}

Mathis and Benoist lab home page:

http://www.joslinresearch.org/PINET/InvestigatorDetail.asp? InvestigatorlD=11 
NF- $\kappa \mathrm{B}$ target genes. The expression of interleukin- $1 \alpha$ (IL-1 $\alpha), \mathrm{IL}-1 \beta$ and COX2, which are normally induced by LPS-mediated activation of macrophages, was inhibited by SB202190. Interestingly, treatment with LT inhibited the expression of the same genes.

The authors conclude that, through phosphorylation of an asyet-unidentified target, $\mathrm{p} 38$ synergizes with NF- $\kappa B$ to induce the expression of anti-apoptotic genes in macrophages. By inhibiting the activation of $\mathrm{p} 38, B$. anthracis changes a macrophage activation signal into a signal for cell death, so enabling the bacterium to evade the innate immune response. ORIGINAL RESEARCH PAPER Park, J. M. et al. Macrophage apoptosis by anthrax lethal factors through p38 MAP kinase inhibition. Science 297, 2048-2051 (2002)

FURTHER READING Guidi-Rontani, C. The alveolar macrophage: the Trojan horse of Bacillus anthracis. Trends Microbiol. 10, 405-409 (2002) WEB SITE

Michael Karin's lab:

http://medicine.ucsd.edu/pharmaco/mkarin.html

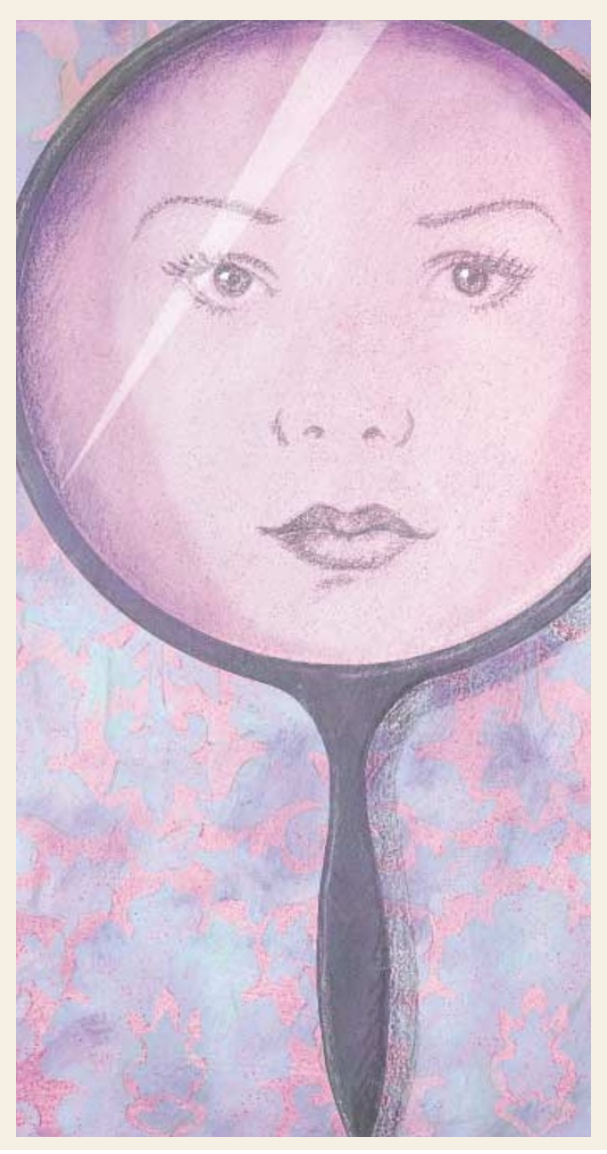

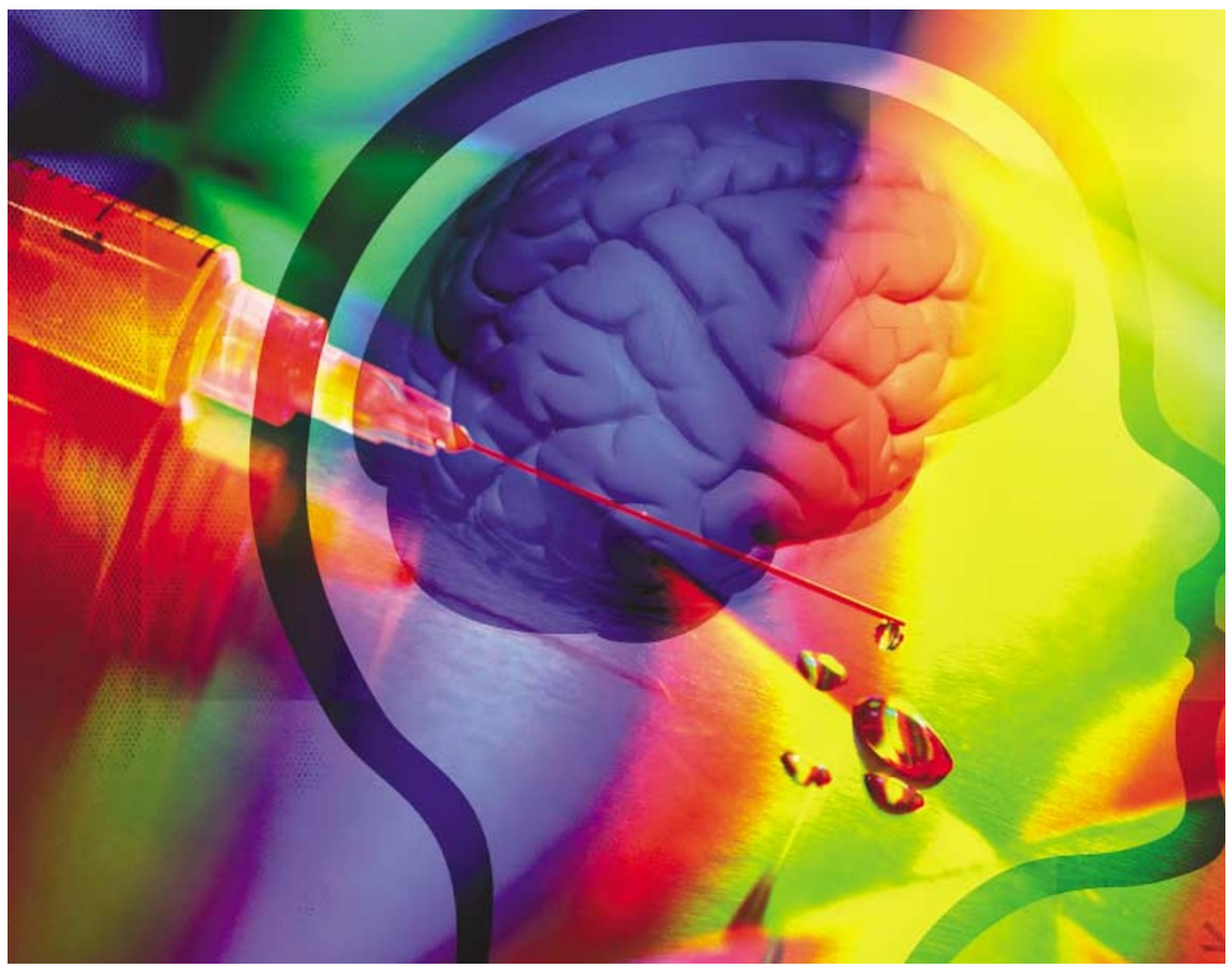

VACCINES

\section{Vaccine revisited}

Earlier this year, Elan Pharmaceuticals and Wyeth-Ayerst were forced to halt Phase II studies of their vaccine for Alzheimer's disease after the discovery that 15 patients had developed severe brain inflammation. The vaccine - a fragment of amyloid precursor protein (APP) known as $\mathrm{A} \beta_{42}$, which stimulates a response to the $\beta$-amyloid plaques that are a hallmark of the disease - had shown highly promising results in preclinical models and Phase I trials.

Two studies in Nature Medicine now indicate that there could still be hope for this strategy after all. Nitsch and colleagues detected a positive antibody response in a subset of patients who took part in the ill-fated trial, and McLaurin and colleagues show that refining the epitope might eliminate the side effects.

Nitsch and colleagues found that serum antibodies from most patients treated with the vaccine plus booster recognized $\beta$-amyloid plaques, diffuse $A \beta$ deposits and vascular $\beta$-amyloid in the brain blood vessels of transgenic mice bred to develop marked Alzheimer's-like $\beta$-amyloid deposits. Importantly, the antibodies did not cross-react with APP, which is found in the nerve cells of both healthy subjects and patients with Alzheimer's disease. In other words, the vaccine selectively induced the desired immune response against disease-associated forms of $\mathrm{A} \beta$ - whether it can prevent cognitive decline will be the focus of future studies.

The study by McLaurin and colleagues assessed whether the beneficial effects of the vaccine could be separated from the inflammatory side effects. Mass spectrometry showed that therapeutic antibodies raised against $A \beta_{42}$ recognized an epitope defined by residues 4 to 10 (termed $\mathrm{A} \beta_{4-10}$ ). Incubating serum that contains antibodies raised against $A \beta_{42}$ with cells was used to show that these antibodies can inhibit both the generation of fibrils (the long thread-like aggregates of misfolded proteins that are associated with the formation of amyloid plaques) and cytotoxicity. When the immune response that is induced by $A \beta_{4-10}$ was assessed in mice, the researchers found that $A \beta_{4-10}$ stimulates a Thelper $2\left(\mathrm{~T}_{\mathrm{H}} 2\right)$ response and the production of anti-A $\beta_{4-10}$ antibodies. But, $\mathrm{T}_{\mathrm{H}} 1$ responses, which many speculate were the cause of the adverse side effects in the clincial trial, were not detected.

These results indicate that a more refined vaccine, based on $A \beta_{4-10}$, might be effective and safe in humans. And, intriguingly, a clearer understanding of the antibody-antigen interactions might lead also to the generation of small-molecule drugs that mimic the effects of the vaccine.

Associate Editor (News), Nature Reviews Drug Discovery

(2) References and links

ORIGINAL RESEARCH PAPERS Hock, C. et al. Generation of antibodies specific for $\beta$-amyloid by vaccination of patients with Alzheimer disease. Nature Med. 15 October 2002

(DOI 10.1038/nm783) | McLaurin, J. et al. Therapeutically effective antibodies against amyloid- $\beta$ peptide target amyloid- $\beta$ residues $4-10$ and inhibit cytotoxicity and fibrillogenesis. Nature Med. 15 October 2002 (DOI 10.1038/nm790)

FURTHER READING Schenk, D. Amyloid- $\beta$ immunotherapy for Alzheimer's disease: the end of the beginning. Nature Rev. Neurosci. 3, 824-828 (2002) 